\title{
APHID-AUTOEPG SOFTWARE FOR ANALYSING ELECTRICALLY MONITORED FEEDING BEHAVIOUR OF APHIDS
}

\author{
TONI PRÜFER ${ }^{1}$, THOMAS THIEME1,*, and W. FRED TJALLINGII² \\ ${ }^{1}$ BTL Bio-Test Labor GmbH Sagerheide, RG Phyto-Entomology, D-18190 Gross Lüsewitz, Germany; 2 EPG Systems, \\ NL-6703CJ Wageningen, The Netherlands \\ * Corresponding author: tt@biotestlab.de, Tel: +49 38209 499561, Fax: +49 38209878762
}

\begin{abstract}
Aphid-AutoEPG is a new software program, developed to acquire and automatically analyse electrical penetration graph (EPG) signals used for monitoring plant penetration by aphids. Characteristically EPG signals include waveforms that allow one to study the effects of plants on the acquisition and transmission of viruses, plant protection substances on pests and identify the location of resistance in plants to aphids. The success of electrical monitoring arises from the fact that different species of aphids generate similar EPG patterns. But the visual analysis of EPG waveforms in recorded signals is very time consuming, which has greatly hampered the wider application of EPG technology. To overcome this Aphid-AutoEPG software was developed, which has been extensively tested using data files for several different species of aphids, M. persicae, A. frangulae gossypii, M. albifrons, S. avenae, B. brassicae and N. ribis-nigri. The results have also been compared with visual analyses using Stylet+ software. When used in combination with the user friendly correction options Aphid-AutoEPG is not only easy to use but can also result in a considerable saving in terms of time.
\end{abstract}

Keywords: feeding behaviour, electrical penetration graph (EPG), software, automatically analyse

\section{Introduction}

The feeding behaviour of aphids is now largely studied using the electrical recording technique originally developed by McLean and Kinsey (1964) and improved by Tjallingii (1978). The recorded signal, which is known as the electrical penetration graph (EPG), provides information on the movements and location of the stylets of aphids in plant tissues and is used to identify the feeding activities of aphids. The different EPG patterns for aphids were defined by Tjallingii (1988) in terms of their amplitude and frequency. Characteristically EPG signals include: 1) a stylet pathway phase, waveforms A, B, C and pd (lumped as $\mathrm{C}$ ), which record intercellular stylet penetration, stylet sheath salivation (A, B and C) and brief $(5 \mathrm{~s})$ cell punctures (pd); 2) a phloem phase, waveforms E1 and E2, which record salivation into phloem sieve elements and passive ingestion of sieve element sap with concurrent salivation, respectively; 3 ) waveform $\mathrm{F}$, recording abnormal stylet mechanics (penetration difficulties); and 4) a xylem phase, active xylem sap ingestion (drinking; Tjallingii 1978, 1988). This knowledge, which was obtained over the last three decades, makes EPG an often used tool in investigating aphid-plant interactions, especially plant resistance mechanisms and virus transmission.

EPGs are obtained by recording (generally with a sampling rate of $100 \mathrm{~Hz}$ ) the electrical signals using a computer and an A/D converter, which are stored in 'raw data files'. Recently, software was developed by several researchers working in this field for distinguishing
70-100 EPG parameters of visually analysed EPG signals (see www.EPGsystems.eu, page downloads), which provides more detailed information on the effects of (1) plants on plant virus transmission (Fereres and Collar 2001; Tjallingii and Prado 2001), (2) plant protection substances on pests (Nisbet et al. 1993) and (3) the location of aphid resistance in (host) plants (van Helden and Tjallingii 1993; Caillaud et al. 1995; Alvarez et al. 2006). The success of this electrical monitoring technique arises from the fact that EPG waveforms and the details of the patterns within these waveforms recorded for different species of aphids are very similar. However, the visual analysis of EPG waveforms is very time consuming, which prevents its wider application. There have been a number of attempts to automate EPG waveform recognition using Fourier processing (Tjallingii 1988; Rahbe et al. 1995) but these have proved unsatisfactory. The major problem is the variability in the signals in terms of the species analysed and quality of the data recorded. Therefore, the obligatory 'on screen' identification of EPG waveforms in the records of signals in raw data files is the 'bottle-neck' in the analyses. To overcome this we developed an EPG software package for use with PCs, which automatically recognizes the different waveforms in the raw data acquired by Aphid-AutoEPG or Tjallingii's software 'Stylet' for PC's. The motivation for developing Aphid-AutoEPG is to reduce the time-consuming manual analyses and in so doing decrease the incidence of human errors occurring during waveform labelling over periods of many hours and improve the EPG-technique. 
We tested Aphid-AutoEPG using EPG recordings of several different species of aphids (e.g. Myzus persicae, Aphis frangulae gossypii, Macrosiphum albifrons, Sitobion avenae, Brevicoryne brassicae and Nasonovia ribis-nigri).

The results of the analysis obtained using AphidAutoEPG software are very comparable to those obtained using Stylet+ software. Automatic analyses using Aphid-AutoEPG followed by a manual check using the friendly correction option results in a considerable saving of time.

\section{Material and Methods}

\section{Hardware Requirements}

Aphid-AutoEPG may be used on all PCs equipped with a minimum screen resolution of $1024 \times 768$ pixels, $1 \mathrm{~GB}$ RAM and at least a $1.5 \mathrm{GHz}$ Single-Core-Processor, but better still with a Dual-Core each of $2 \mathrm{GHz}$ or more. For the acquisition of raw data there is an especial need for the DC-EPG-system "Giga-8" and data-logger "DATAQ DI-710".

\section{Software Requirements}

To run Aphid-AutoEPG the computer should have Windows XP or higher as the operating system. Re- quired frameworks, which are automatically installed if the internet connection is active, are Microsoft.NET Framework $3.5+$ service pack 1 and Microsoft XNA Framework 3.0. For the acquisition of data the driver required for the data-logger is WinDAQ Data Acquisition software, DATAQ Instruments ActiveX Controls and WinDaq Add-on package from DATAQ Instruments.

\section{Results}

\section{Programming}

Aphid-AutoEPG was developed using C-Sharp programming language in a NET 3.5 Framework. Visual Studio 2008 was the integrated development environment. Each computer language is especially useful for a specific aim, for instance, FORTRAN is often used for numeric calculations and Java for the development of platform independent applications. Several important factors were taken into consideration during the development. In particular, the diverse nature of the calculations, efficient management of memory because of the large volume of data, the graphic presentation of curves and the design of a simple graphical user interface.

Aphid-AutoEPG facilitates not only the analysis of EPG recordings acquired independently by different sys-

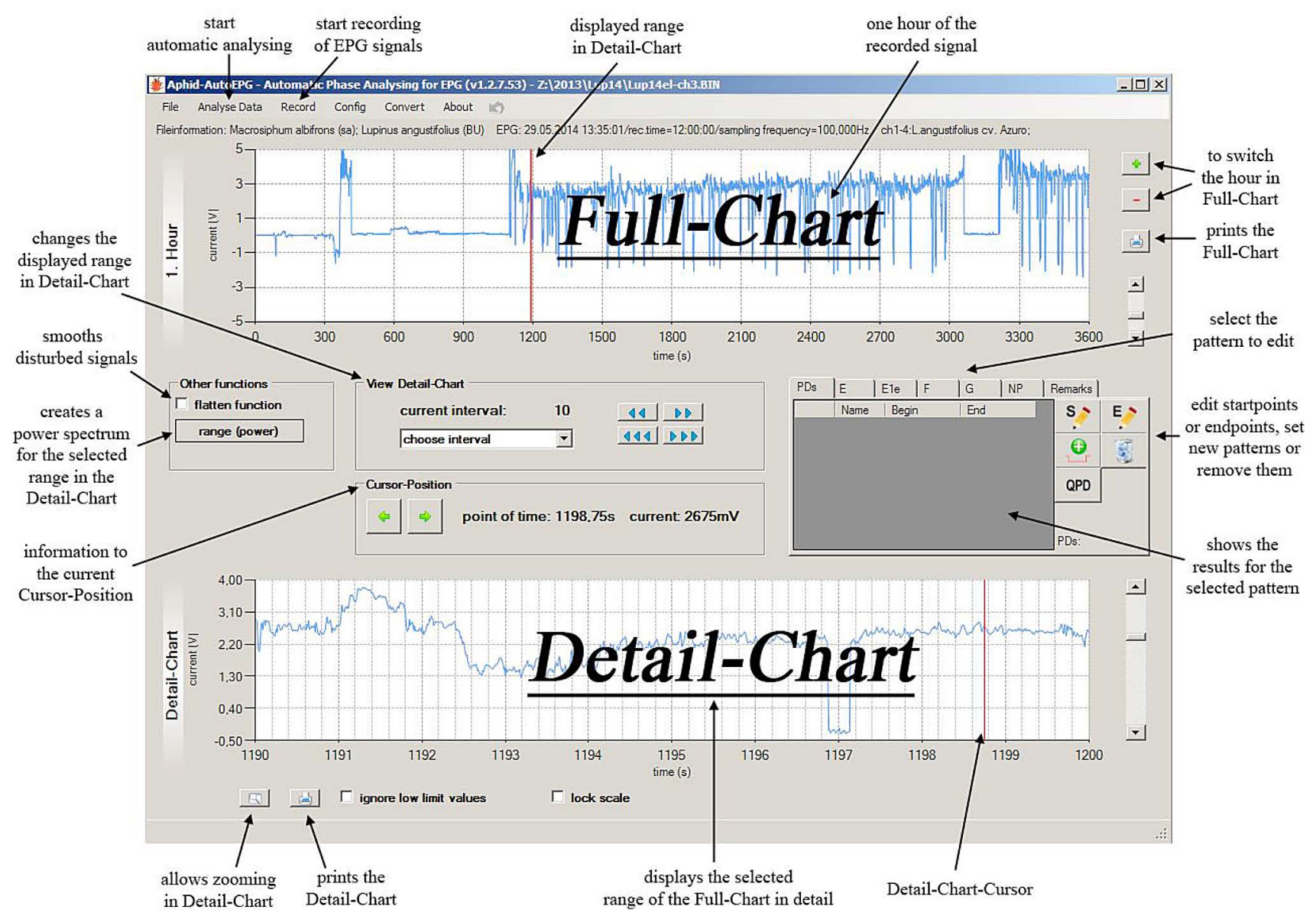

Fig. 1 View of the working window of Aphid-AutoEPG before the start of an automatic analysis. 
tems, but also the acquisition of raw data. This software can read EPG raw data acquired by Tjallingii's system for use with PCs. This data is normally stored in files each of which contains an hour long section of the recording. Aphid-AutoEPG offers an additional file format that enables one to store all the data in one file.

Collaboration between BTL and Freddy Tjallingii was initiated in order to improve this software.

\section{Description of the Aphid-AutoEPG Program}

Aphid-AutoEPG is user-friendly and menu-driven software. The main menu allows the user to specify the type of work: e.g. recording/acquisition of new EPG data; automatic analyses and conversion (switching between the different types of data) of EPG raw data files.

The screen shows 3 areas: (1) 'Full-Recording', a graphics zone displaying an one hour overview of the whole signal (EPG) in a particular file; (2) 'Detailed-Recording', the working area displays in more detail a selected part of the signal on which the user can correct the waveforms after the automatic analyses; (3) a working area (between Full-Recording and Detailed-Recording) with numerous editing functions and information on co-ordinates of the cursor position and results of the automated analyses of particular sections of EPG data (Fig. 1).

A

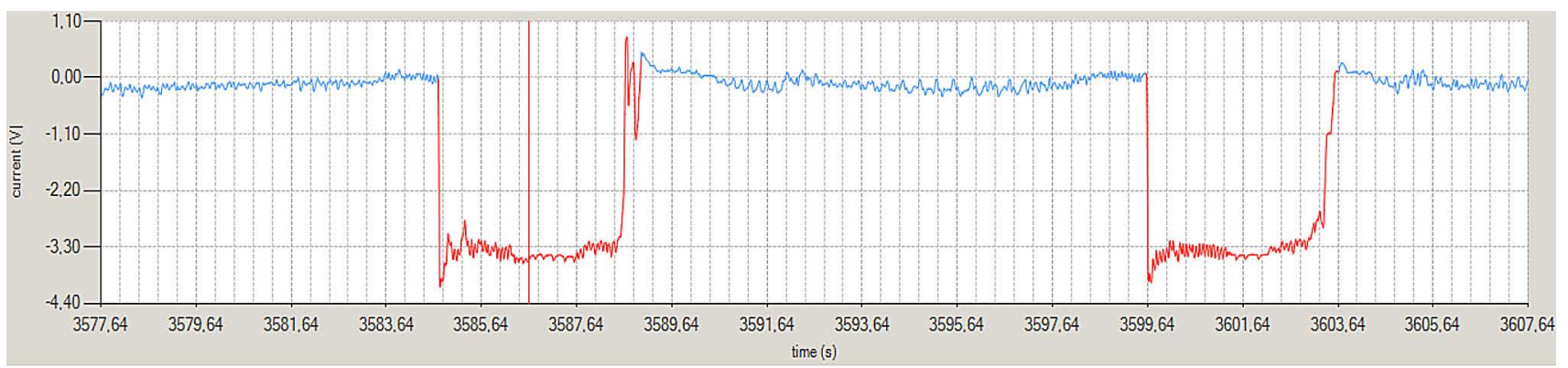

B

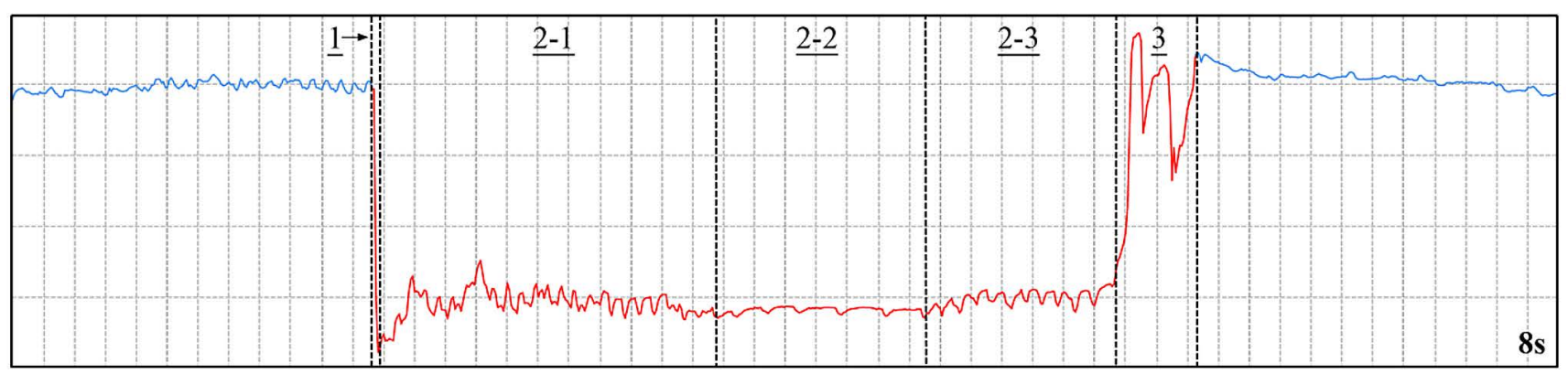

C

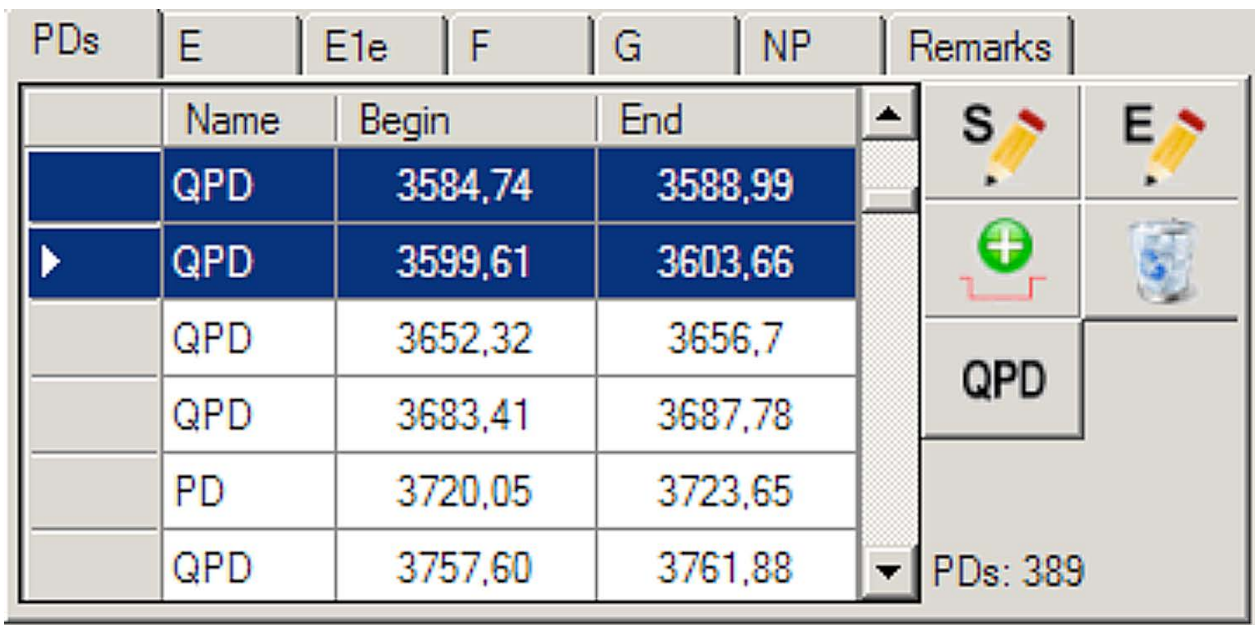

Fig. 2 Example of the results of an automatic analysis of data using Aphid-AutoEPG. A Full recording showing two PD's (highlighted in red) in the signal recorded between the 3577.64 and 3607.64 second; B Detailed recording showing eight seconds of the recording of a single PD, which is further qualified PD (QPD) as it contains records of three phases with corresponding sub-phases; $\mathbf{C}$ window shows selected results (here partly those for PD's). 
The part of the signal displayed on the Detailed-Recording is indicated on the Full-Recording by means of a moveable cursor. The user has several options for changing the signal displayed on both charts. Moving the Full-Recording cursor triggers a change in the section of the Detailed-Recording displayed. It is also possible to change the length of the section of the Detailed-Recording displayed or move it a few seconds forwards or backwards in time. Moreover the Detailed-Recording has a zooming function, which can be used to enlarge a particular selection and so view it in more detail. In addition, the user is able to print both charts. These printouts automatically include further information on the EPG recording being studied.

There is no need for the user to identify and label the waveforms in the recording because this is done automatically by Aphid-AutoEPG. The results are presented on both recordings and in a table in the working area (Fig. 2). The six buttons correspond to the different EPG patterns defined by Tjallingii (1990). The pattern appended to the EPG becomes immediately visible on both recordings and the type and position in time of all the start- and endpoints can also be displayed on screen or printed in tabular form. To check the results of the automatic analyses the user has to select a specific pattern in the results table, which results in the corresponding waveform being highlighted in red. After having confirmed it is the correct pattern and its start and endpoint these results can be saved. If the user wishes to correct the results this is easily done by clicking the results table and editing the selected pattern on Detailed-Recording in terms of its start- or endpoint by a simple click of the mouse at the desired position in time, change the pattern, add a new or delete an identified pattern.

After checking/editing all patterns (or at any time during this operation) the user can save the results (type, time position and voltage of the signal) for subsequent calculation of the EPG parameters or for transfer to spreadsheets or software for statistical analyses and graphical presentation.

The success of the software in identifying the patterns depends on the quality of the data. If it consists of weak signals with minor changes in voltage the identification is unreliable. But this is also the case if the analysis is done by visually scanning the recording. The quality of raw data is dependent on the aphid, as each species of aphid is not similarly suitable because it is more difficult to attach a wire to very small individuals and the electrical conductivity of those with a thick waxy covering is poor. In addition, the patterns in waveforms can be species specific; the signals recorded from aphids transferred to non-host plants can be relatively weak; disturbance by radiation from other pieces of equipment if the Faraday cage is not installed correctly; the experience and patience of the user during the preparation and execution of an experiment can also markedly affect the quality of the data recorded.
The automated analyses of a 12 hour recording will take approximately 30 to 60 seconds, depending on the number of phases and the performance of the PC. The duration of the subsequent checking, if thought necessary, depends on the experience of the user. But because each pattern and its duration (start- and endpoint) are in the results table and highlighted in red in the displayed charts it is easy to judge and edit the results of automated analyses (Fig. 2). Using Aphid-AutoEPG it is possible to directly select each event within a pattern of interest in the results table and display it. In total up to 20 minutes can be scheduled for a complete verification of results of a 12 hour run (including the checking of the patterns of more than 200 pd's).

During automated identification it is nearly impossible to misidentify waveform F (Fig. 3A). This only occurred if this phase was shorter than 10 seconds. Occasionally this phase is displayed in smaller fragments. In such cases it is easy to use the "join" function. The waveform $G$ is never misidentified (Fig. 3B). The identification of the phloem phase E1 and E2 is more difficult (Fig. 3C and D). Whereas misidentifications are very rare the start- or endpoints are not always determined precisely. The waveform E1e (watery salivation at extracellular voltage level) is not identified by Aphid-AutoEPG and its start- and endpoints have to be determined visually. Identification of non-penetration (np, Fig. 3D) by the automated analysis is in most cases correct. Usually the signal for $\mathrm{np}$ is slightly above or below $0 \mathrm{~V}$ within the interval $-0.05 \mathrm{~V}-0.05 \mathrm{~V}$. However, there are few recordings with greater fluctuations in intensity $(-0.2 \mathrm{~V}-0.2 \mathrm{~V})$, which require editing. Identification of the waveform pd (brief intracellular stylet punctures, Fig. 4) is in most cases correct. Exceptions occur when the quality of the signal is very poor because of its low voltage (below $0.2 \mathrm{~V}$ ). In this case Aphid-AutoEPG offers the possibility of reducing the threshold in the main menu "Config". As mentioned before the patterns can be species specific. Aphid-AutoEPG is able to compensate for this and display the waveform patterns for different species of aphids (e.g. of pd's of four species of aphids see Fig. 4).

\section{Additional Facilities}

Aphid-AutoEPG offers an extensive recording function with greater scope of operation. This makes it easy to calibrate the signal at the start of a recording lasting several hours. This software has a new file format ( ${ }^{*}$.bin) that enables the user to store the data for a complete recording in a single file. It is also possible to print the charts displayed along with all the important information on the functions used. With the help of these different options the user can edit the automated analyses and improve the quality of poor recordings.

An additional module is under development that will enable Aphid-AutoEPG to control experiments: arrange a project, including the corresponding EPG runs; store the results and carry out automated analyses; statistically 
A

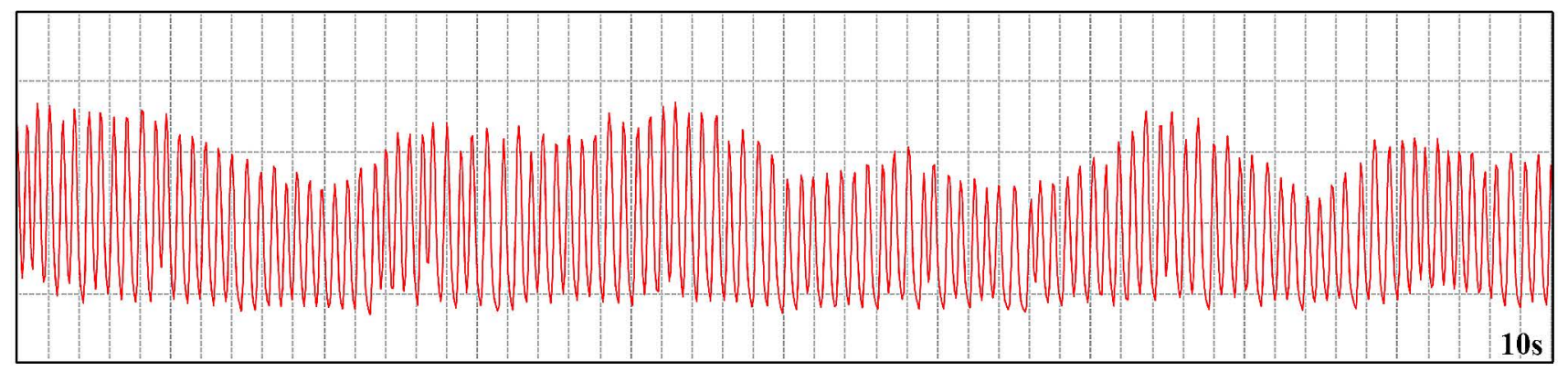

B

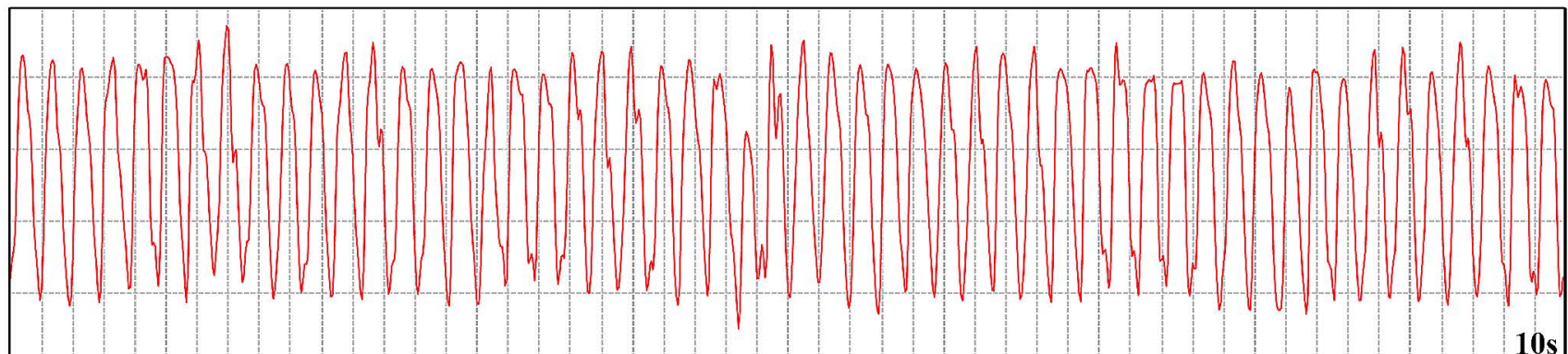

C

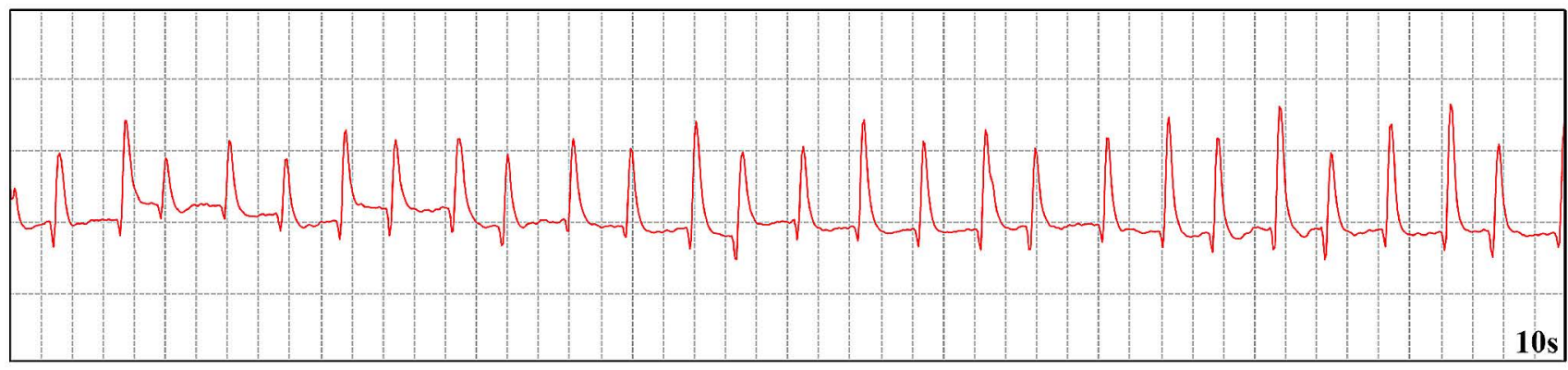

D

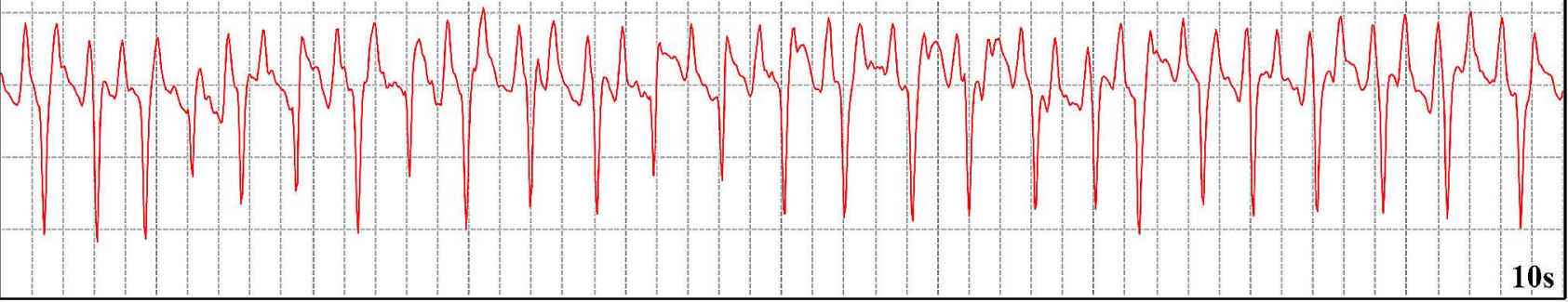

E

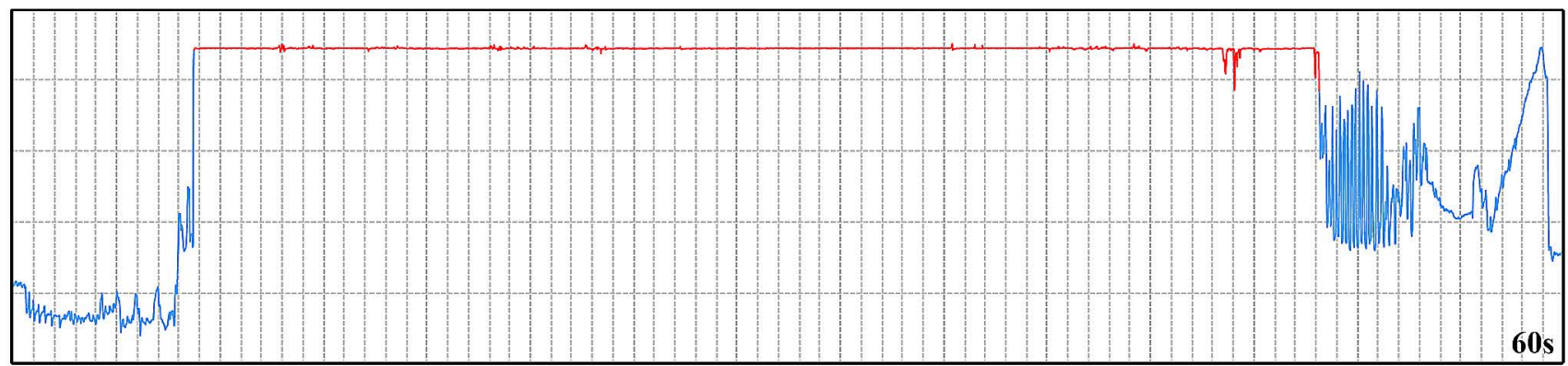

Fig. 3 Examples of recordings automatically acquired and analyzed using Aphid-AutoEPG. Ten second recordings of the $F$ pattern (A), G pattern (B), E1 pattern (C), E2 pattern (D) and a 60 second recording of a non-penetration phase (np highlighted in red) (E). The scales of both axes ( $x$ time and $y$ current) are not shown. 
A

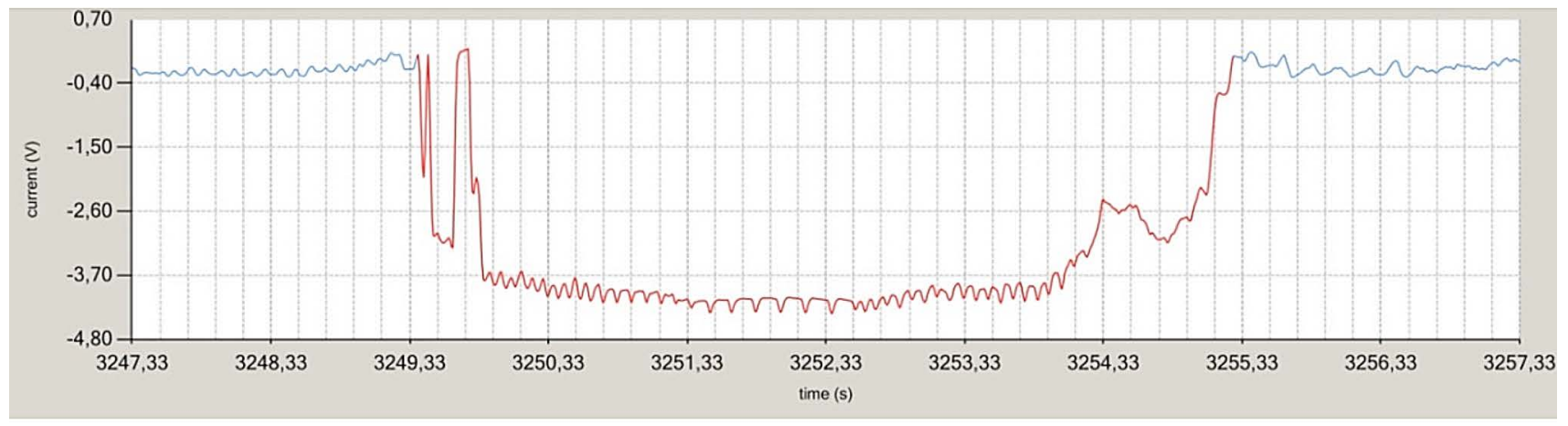

B

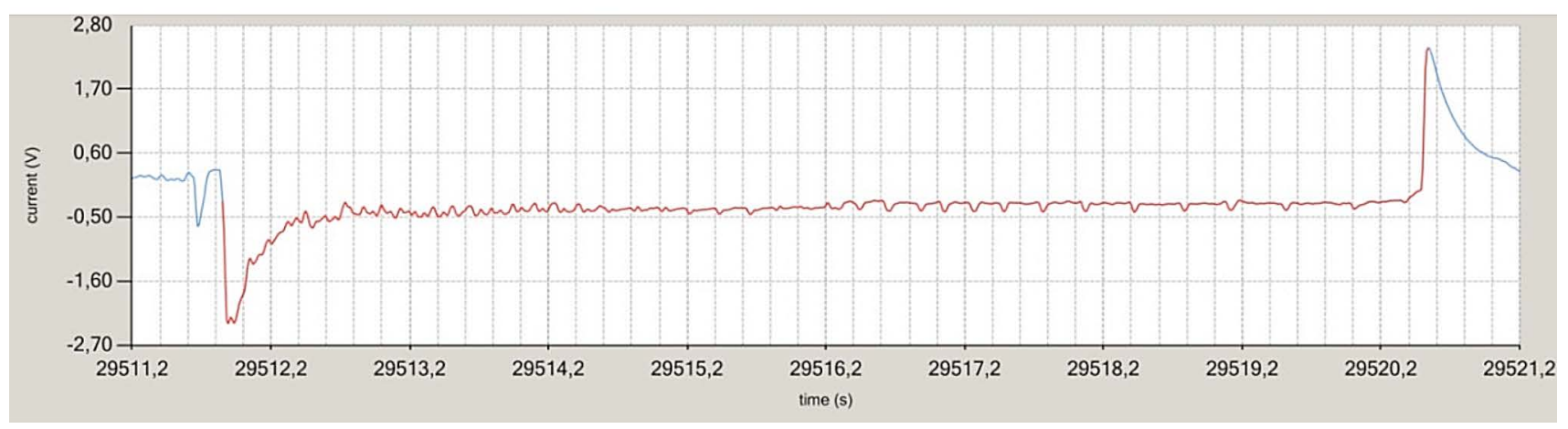

C

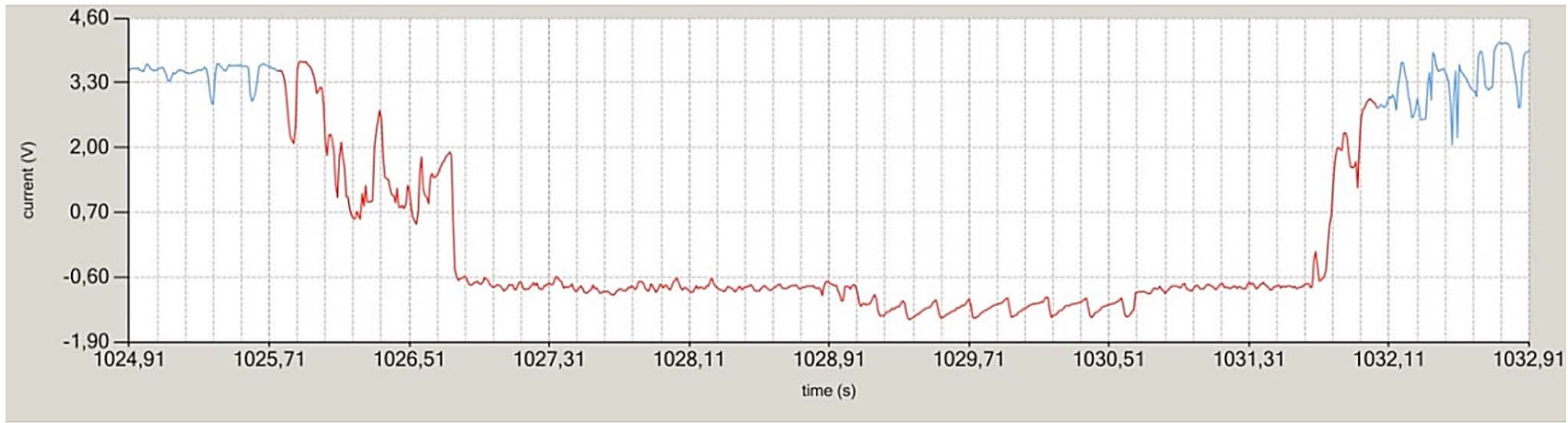

D

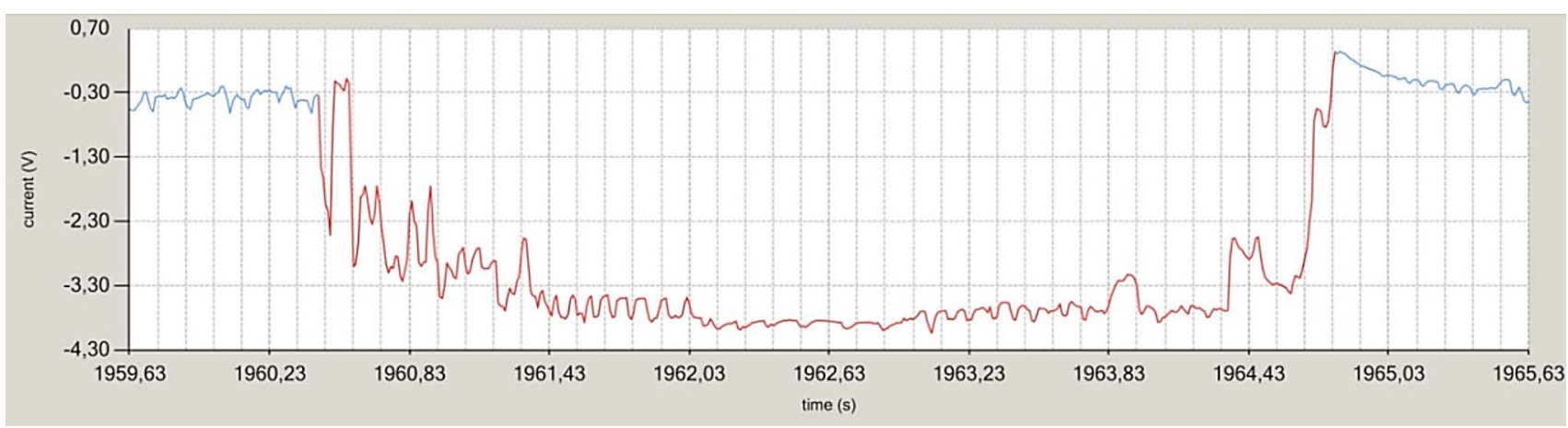

Fig. 4 Species specific patterns of the start and endpoints of the potential drops (PD) automatically identified without manual correction for: A Aphis frangulae gossypii, indicates mostly nearly perfect PDs; B Brevicoryne brassicae, often indicates a weaker signal in the later phases of a recording caused by aphid wax production; C Macrosiphum albifrons, typical rectangular wave in sub-phase 2 of phase 2 is more triangular; D Myzus persicae, easily recognizable patterns in all phases. 
analyse and store EPG data; store all data in a central data base so that it can be accessed by all the authorized computers in a network. This will have the following advantages: fast and easy management of centrally stored data; separate EXCEL-files for statistical analyses; well-arranged presentation of projects and their results; support for users with different needs (e.g. automatically generated protocols for experiments and different possibilities for filtration over all projects); keeping all in one file from recording of raw data to its statistical analysis.

Aphid-AutoEPG is completely compatible with Tjallingii's software 'Stylet+' for PC's. Raw data and results recorded using Stylet+ can be used with our software and vice versa.

\section{Conclusion}

Aphid-AutoEPG is a new software program, developed to acquire and automatically analyse electrical penetration graph (EPG) signals used for monitoring plant penetration by aphids. Characteristically EPG signals include waveforms that allow one to study the effects of plants on the acquisition and transmission of viruses, plant protection substances on pests and identify the location of resistance in plants to aphids. The success of electrical monitoring arises from the fact that different species of aphids generate similar EPG patterns. But the visual analysis of EPG waveforms in recorded signals is very time consuming, which has greatly hampered the wider application of EPG technology. To overcome this Aphid-AutoEPG software was developed, which has been extensively tested using data files for several different species of aphids, $M$. persicae, A. frangulae gossypii, $M$. albifrons, S. avenae, $B$. brassicae and $N$. ribis-nigri. The results have also been compared with visual analyses using Stylet+ software (W. F. Tjallingii, EPG systems). When used in combination with the user friendly correction options Aphid-AutoEPG is not only easy to use but can also result in a considerable saving in terms of time.

\section{Acknowledgements}

The authors would like to thank Prof. A. F. G. Dixon from UEA Norwich for useful discussions, and Jörn Reck from BTL for his advice on various practical aspects of IT.

\section{REFERENCES}

Alvarez AE, Tjallingii WF, Garzo E, Vleeshouwers V, Dicke M, Vosman B (2006) Location of resistance factors in the leaves of potato and wild tuber-bearing Solanum species to the aphid Myzus persicae. Entomol Exp Appl 121: 145-157.

Alvarez AE, Broglia VG, D'Amato AMA, Wouters D, van der Vossen E, Garzo E, Tjallingii WF, Dicke M, Vosman B (2013) Comparative analysis of Solanum stoloniferum responses to probing by the green peach aphid Myzus persicae and the potato aphid Macrosiphum euphorbiae. Insect Sci 20: 207-227.

Caillaud CM, Dedryver CA, Di Pietro JP, Simon JC, Fima F, Chaubet B (1995) Clonal variability in the response of Sitobion avenae (Homoptera: Aphididae) to resistant and susceptible wheat. Bull Entomol Res 85: 189-195.

Fereres A, Collar JL (2001) Analysis of noncirculative transmission by electrical penetration graphs. In: Harris KF, Smith OP, Duffus JE (eds) Virus-insect-plant interactions. Academic Press, San Diego, CA, USA, pp. 87-109.

Le Roux V, Dugravot S, Campan E, Dubois F, Vincent C, Giordanengo P (2008) Wild Solanum resistance to aphids: antixenosis or antibiosis? J Econ Entomol 101: 584-591.

McLean DL, Kinsey MG (1964) A technique for electronically recording aphid feeding and salivation. Nature 202: 1358-1359.

Nisbet AJ, Woodford JAT, Strang RHC, Connolly JD (1993) Systemic antifeedant effects of azadirachtin on the peach-potato aphid Myzus persicae. Entomol Exp Appl 68: 87-98.

Rahbé Y, Febvay G, Delobel B, Bonnot G (2000) Amino acids and proteins as cues in interactions of aphids (Homoptera: Aphididate) and plant. In: Walker GP, Backus EA (eds) Principles and applications of electronic monitoring and other techniques in the study of homopteran feeding behavior. Thomas Say Publications in Entomology, Entomological Society of America, Lanham, USA, pp. 212-236.

Tjallingii WF (1978) Electronic recording of penetration behaviour by aphids. Entomol Exp Appl 24: 721-730.

Tjallingii WF (1988) Electrical recording of stylet penetration activities. In: Minks AK, Harrewijn P (eds) Aphids, Their Biology, Natural Enemies and Control. Elsevier, Amsterdam, The Netherlands, pp. 95-108.

Tjallingii WF (1990) Continuous recording of stylet penetration activities by aphids. In: Campbell RK, Eikenbary RD (eds) Aphid-Plant Genotype Interactions. Elsevier, Amsterdam, The Netherlands, pp. 89-99.

Tjallingii WF (2006) Salivary secretions by aphids interacting with proteins of phloem wound responses. J Exp Bot 57: 739-745.

Tjallingii WF, Prado E (2001) Analysis of circulative transmission by electrical penetration graphs. In: Harris KF, Smith OP, Duffus JE (eds) Virus-insect-plant interactions. Academic Press, San Diego, CA, USA, pp. 69-85.

Van Helden M, Tjallingii WF (1993) Tissue localisation of lettuce resistance to the aphid Nasonovia ribisnigri using electrical penetration graphs. Entomol Exp Appl 68: 269-278.

Will T, Van Bel AJE (2006) Physical and chemical interactions between aphids and plants. J Exp Bot 57: 729-737. 